Voix et Images

voixetimages

\title{
Pour Gauvreau : un témoignage
}

\section{Janou Saint-Denis}

Volume 3, numéro 1, septembre 1977

Nicole Brossard

URI : https://id.erudit.org/iderudit/200086ar

DOI : https://doi.org/10.7202/200086ar

Aller au sommaire du numéro

\section{Éditeur(s)}

Les Presses de l'Université du Québec

\section{ISSN}

0318-9201 (imprimé)

1705-933X (numérique)

Découvrir la revue

\section{Citer cet article}

Saint-Denis, J. (1977). Pour Gauvreau : un témoignage. Voix et Images, 3(1),

32-39. https://doi.org/10.7202/200086ar d'utilisation que vous pouvez consulter en ligne.

https://apropos.erudit.org/fr/usagers/politique-dutilisation/ 


\section{Pour Gauvreau : un témoignage}

La poésie m'a toujours envoûtée et je suis définitivement amoureuse de chaque poète réellement perçu et vase communiant. Fidèle par surcroît, JAMAIS JE NE TRAHIRAI Antonin Artaud, Henri Michaux, Maïakowski et Claude Gauvreau. Par je ne sais quelles affinités secrètes, je me sens une appartenance à cette lignée d'audacieux. Je vous dois une petite explication ou comme vous dites si bien cune justification». Une attitude aussi irrationnelle est dénuée de logique et loin du formalisme à la mode! Je vous invite donc à un recul de plus de vingt-cinq ans dans cet espace devenu le vôtre où bien des choses ont changé sauf l'espace du dedans d'ici.

Mon prénom Janou, je le dois à Claude qui me baptisa à l'affreux vin Québérac sur les fonts de la Hutte suisse rue Sherbrooke en l'an 1950. Huit ou neuf années plus tard, un de ces dimanches engourdis de l'hiver québécois, j'improvise (pour tromper l'ennui de notre jeunesse) ou plutôt j'inaugure au café El Cortijo rue Clark à Montréal, des lectures de poèmes d'ici et d'ailleurs (deux heures d'affilée et cela a duré tout un hiver) agrémentées par les premières chansons et la guitare de Tex, l'un des peintres assidus de notre bohème ${ }^{2}$. Je me risque un soir à leur lire des extraits de Brochuges, première publication de Claude (1956). Sans effort je me rappelle l'état délirant et la luminescence persistante qui m'envahirent alors, ne me laissant plus de répit dans ce besoin suscité d'absolu, de beauté, de grandeur, d'ascension spirituelle et c'est avec une passion sans limites et un amour indéfinissable que je décide de mettre en scène pour le Centre d'essai à l'école des Beaux-Arts, deux courtes pièces de Claude Gauvreau choisies parmi les cinquante-deux écrits qu'il me présenta pour en faire une copie dactylographiée. Le choix n'était pas simple... chacun de ces objets poétiques fusant d'une intensité rare... mais j'étais limitée par un budget plus que restreint pour ne pas dire inexistant et le peu de technicité mise à ma disposition. J'optai dans le cadre d'un spectacle pour: la Jeune Fille et la lune et les Grappes lucides, acceptés sans difficultés par monsieur Nathan Karczmar, directeur du centre d'essai. Le public fut fort impressionné par la réalisation scénique de la jeune fille et la lune, monologue dont j'étais l'unique interprète. Un grand panneau peint de bleus et de verts, un 
filet de pêche, des banderolles de papier froissé simulant les algues, sous un éclairage d'eaux et de remous surgissait une fantasmagorie sousmarine que j'avais entièrement conçue et réalisée conjointement avec les élèves de l'école des Beaux-Arts pour ce long poème dramatisé, qui était une sorte d'adaptation à l'univers gauvresque et à celui des Québécois, de «la Ralentie" d'Henri Michaux, poète alors inconnu à Montréal, sauf par quelques rares personnes ayant entendu le disque enregistré magistralement par Germaine Montero.

S'agissait-il de réminiscences? Claude avait certainement lu'les ceuvres de Michaux parues à cette époque... il dévorait les livres à un rythme étonnant (les faisant directement venir d'Europe) souvent avant même que nous nous doutions de l'existence de tel auteur... comme le drogué sans cesse à la recherche d'une nouvelle drogue plus soutenue! De toutes manières, je n'y ai vu aucun plagiat, plutôt comme cela naît dans le jazz, une sorte d'improvisation sur un thème offrant une riche gamme de variations. En pleine noyade, une femme avant de quitter cette existence, enregistre à voix haute des images brèves, des émotions déchirantes, des souvenirs hachés, des paysages internes ou environnants, des assauts, des fuites, des amours vécues ou rêvées. La Jeune Fille et la lune, c'est l'effarante solitude devant la Mort.

La deuxième pièce retenue, les Grappes lucides, c'est autre chose que je définis aujourd'hui comme une projection fœtale du splendide monument funéraire que Claude Gauvreau s'est élevé avec Les oranges sont vertes. Le personnage principal des Grappes lucides, un dénommé Saplerbe est à la recherche soutenue de sa COULEUR... couleur aperçue et disparue... il croit la trouver un moment dans l'amitié avec Guyaldys, puis dans l'amour avec la femme Idmaf lorsque surgit des eaux une sirène qui ne réussit qu'à l'entraîner à sa suite et Saplerbe marchera longtemps serein et confiant dans la mer vers sa Couleur, la ligne d'infini humainement inaccessible dans laquelle d'ailleurs il s'efface à l'œil. Le faible apport anecdotique dans les Grappes lucides n'appauvrit pas la tragédie de l'être humain face à la transcendance. La langue sonore, vibrante de tous les cris, détachée de toutes contingences pénètre nos ouies dilatées tel un liquide clarifiant nous obligeant à un abandon inconditionnel aux divagations et aux échappements de l'âme, premières données de l'Infini.

Yvirnig des Oranges sont vertes et Saplerbe des Grappes lucides sortent de la même matière. Yvirnig concrétise l'abstrait Saplerbe, Yvirnig confirme l'existence de l'essence de Saplerbe. Saplerbe est la pensée du poète, Yvirnig est l'existence rationnelle de cette pensée. Mycroft de la Charge de l'orignal épormyable serait alors la marionnette figurant la pensée du poète bousculée, charriée, démantibulée, disséquée par ceux qui l'entourent.

J'étais en Europe lors de la création de la Charge... par le groupe Zéro (1970) sous la direction de Claude Paradis avec la participation totale de Monique Duplanty, Jacques Crête, Albert G. Paquette et d'autres que je ne connais pas. Malgré mon absence, je suis convaincue que ce groupe 
malgré les difficultés survenues au cours des représentations a réalisé une création en parfait accord avec l'auteur parfois aussi étonné qu'eux de leur hardiesse et je n'ignore point qu'à l'instar de tous les pionniers, ils seront trop facilement oubliés et même effacés au profit de l'exploitation sur le plan commercial et rentable, d'une œuvre qui exige l'engagement individuel de tous ceux qui y participent.

Ce qui me paraît le plus important à élucider ici, est d'abord le droit de la poésie au théâtre! et non l'explication concertée de la poésie au théâtre. Je sais des étudiants d'un cégep de Montréal qui pour tenter de saisir la parole de Claude Gauvreau ont cherché la signification mot à mot dans les dictionnaires; séparant l'esprit du corps, ils n'ont touché qu'à l'absurdité de mots vidés de leurs multiples sens et devenus incolores sans leur environnement structuré par le poète. II est plus facile plus expéditif de définir... que de prendre conscience d'une pensée! Au même titre que l'histoire (souvenir) l'art (imagination) est absent de nos écoles. Pour la poésie, manque d'intérêt total dû à une absence totale d'éducation, d'initiation à la parole dans toutes nos institutions.

"Le théâtre n'est pas un miroir réfléchissant, c'est un verre grossissant " déclare Maïakowski dans quatre vers fameux. Lorsque Les oranges sont vertes envahissent la scène du Théâtre du Nouveau Monde, un réalisme à outrance règne sur la dramaturgie québécoise et "seul, répète-t-on, Michel Tremblay qui parle comme tout l'monde communique avec tout I'monde "... quelques nouveaux dramaturges québécois ont mis l'affaire dans leur sac, et tout l'monde s'est mis à faire des pièces de tout l'monde pour tout l'monde. Boff... on lui a fait sa fête à Gauvreau, on peut passer aux choses sérieuses, rentables, quoi... Moi Janou Saint-Denis, je dis qu'on lui a fait sa tête à Gauvreau comme on espère couper la tête à tout le théâtre poétique, à la poésie itou. Et ça c'est grave, lorsque l'on fout la poésie dehors pour y faire entrer sa bédaine flasque et humide et pour y parader son CUL sans ailes, encore mal essuyé de la grosse marde qu'on a évacuée...

\section{Certains diront:}

"que le TNM a obtenu une belle assistance avec Les oranges sont vertes" "que l'monde est venu même s'y comprend rien "

"qu'il vaut aussi bien voir un Gauvreau au TNM plutôt que l'un de ces dramaturges étrangers qui dérangent avec leur vision universelle»

"que Gauvreau ça n'est pas du vrai théâtre québécois parce que le langage est faux et qu'y faut faire un effort de comprenure pour l'atteindre" "qu'on y trouve pas son identification, sa politique, ses piasses, sa cuisine, ses ouètrices pis sa taverne"

"que les prélats domestiqués qui nous ont taré l'esprit au Québec c'est moins important que les hot dog stimés qui nous crispent l'estomac" "que la poésie ça se vend pas pis que le théâtre faut que ça paye"

Après une longue conspiration de silence (1958 à 1970) alors que Claude ne cesse d'écrire et que les comploteurs ne cessent d'étouffer sa voix, on 
se demande par quelle brèche il a pu passer pour apparaître et se mesurer au gros public à cet événement grandiose que fut "la Nuit de la poésie"! Dans le numéro 62 de Vie des arts on peut lire une ligne sur son passage avec les "grands" de notre poésie: "Gauvreau, l'Isidore Isou du Québec". Je connais et je respecte Isou le grand pape du lettrisme, avec lequel j'ai d'ailleurs travaillé. Et je puis vous affirmer que ce sont deux unicités intégrales et que le lettrisme et l'exploréen sont deux recherches complètement distinctes, j'ajouterai même que le poète québécois le plus lettriste est Raoul Duguay lequel épithète Gauvreau de lettriste. Même erreur de la part d'Hubert Aquin. La base du lettrisme est l'implique et le discrèpant, la base de l'exploréen est la réplique et la charge!

Malgré ce malentendu (tout à fait professionnel) quelques personnes ont compris qu'un poète a une manière de dire les choses qui ne livre pas qu'un seul plan de la réalité, mais multiples dominantes de celle-ci que nous ne connaissons que très vaguement et si superficiellement, tentés que nous sommes de les ignorer, à cause de la peur qui nous étreint devant les grandes contradictions de l'Univers: l'amour et la mort, la joie et la douleur, la présence éternelle et l'absence subite. La durée dans la poésie de Claude Gauvreau est à la haute pointe de la rencontre du passé, du présent et du futur. La masse ignore la poésie et les poètes. Les quelques récitations lues au hasard de la scolarité ne sont pas suffisantes pour initier les jeunes et créer un lien profond et solide entre le poète et l'individu. La poésie n'est pas qu'une science du langage - elle est liberté, explosion de l'inconscient, productrice de rêves et génératrice d'une pensée ouverte, éclatée. La poésie unifie par les mots: l'inspiration et l'image, la pensée et l'existence, l'éthique et l'esthétique.

Que des êtres de chair et d'os parlent un langage qui n'est pas celui que l'on entend chaque jour déroute certes - le poète bouscule les mots, les empale, les creuse; il organise les sons pour donner une autre DIMENSION aux cris, aux extases, aux angoisses, aux délires, à l'Amour - le poète est feu d'artifices, volcan des violences, ruisseau gémissant dans sa coulée, âme se tordant sur la bouche, délire joyeux ou trouble ou jouissance cosmique. II nous faut être souple, intelligent, alerte, confiant pour demeurer DISPONIBLE à la parole du poète.

Notre théâtre de cuisine nous paralysera l'âme, si nous ne savons pas nous envoler à temps, nous échapper du tablier et des casseroles pour suivre la fumée qui s'élève toujours vers l'ailleurs... non pas que je souhaite un théâtre d'évadé puisqu'au contraire, je slis persuadée que le théâtre poétique favorise, par sa liberté d'actes et de paroles, une connaissance de soi et du monde.

Le malheur est qu'il est difficile aujourd'hui de trouver un metteur en scène qui au moment de la production, s'identifie entièrement à l'œuvre qu'il théâtralise pour réussir à faire comprendre l'auteur sans avoir à recourir à une plasticité monotone du jeu, à la réduction d'une magnificence du texte par les obsédés de la coupure! Cédant au goût facile du public dont on généralise trop les aptitudes, le metteur en scène simule 
une simplification qui ne contente personne et n'atteint pas le but proposé par l'auteur, la communication par les entrailles.

Les comédiens sont généralement gauches et empruntés dans une œuvre poétique qu'ils interprètent le plus souvent en routiniers du métier, ni plus ni moins. Le plus dur au théâtre, c'est de vivre et non de simuler un personnage. Évidemment, l'acteur ou l'actrice se consume plus rapidement - à lui le choix d'être rare et magnifique - la productivité et le rendement d'un comédien ou d'une comédienne n'ont pas à être calculés de manière compétitive. II est peut-être utopique de croire qu'il existe des acteurs aujourd'hui, capables de brûler une tranche physique de leur existence sur un plateau... il y a eu pourtant un poète-dramaturge qui n'a pas compté les jours qui lui restaient à vivre pour créer par ses textes et ses écrits sur le théâtre, de multiples possibilités libérantes de donner à notre théâtre une consistance et une profondeur, une survie et une aération qui peuvent le mener aux dimensions du Mystère de l'Être.

Le théâtre de Claude Gauvreau n'est pas que poétiques envolées, il est tragique et réel dans toutes ses données parce qu'il magnifie l'être humain et s'il n'évoque pas uniquement l'environnement matériel (dans Les oranges sont vertes l'auteur a signifié lui-même au décorateur l'évolution décadente de l'environnement) il invoque toutes les vibrations cosmiques qui nous sont inhérentes, les ondes magnétiques qui habitent l'espace et provoquent d'étranges secousses dans le cerveau des humains.

Il est temps que le public renonce au confort intellectuel et qu'il ne se contente plus de ces réflexions bâtardes:

"c'est ben toujours ce que j'ai vu et entendu"

«faut dire ça comme ça»

"c'est ben ça, c'est ça qui est ça"

"on m'a toujours dit ça»,

et alors braves gens, à quoi ça sert le théâtre ? à s'enfoncer dans la boue? à se laisser brimer dans l'ignorance la plus totale des choses de l'esprit? qui veut tuer le rêve, le désir de s'initier hardiment à la profonde connaissance des forces insoupçonnées de l'Univers que nous habitons provisoirement? quel beau voyage! pour une fois on peut abandonner le "Ouais, c'est ben ça, j'le savais " pour venir voir et entendre du nouveau, de l'inédit, du spécial, de l'extra. Je suis bien d'accord: il ne faut pas se méprendre sur l'intérêt et la curiosité publique des spectateurs; friands de scandales et de potins sordides, l'beau monde s'est réveillé après tant d'indifférence tel un vautour humant l'odeur du beau cadavre, il est venu en foule remuer ses cendres avant qu'on n'en parle plus et qu'il ne soit plus à la une de la nécrologie! Dès le lendemain; les penseurs poltrons et les panses culottées n'ayant plus la bête aberrante devant eux, ayant eux-mêmes participé à sa destruction, se sont mis à décortiquer leur trouille, leur peur car: «Si fort que nous réclamions la magie, nous avons peur au fond d'une vie qui se développerait tout entière sous le signe de la vraie magie." (Antonin Artaud) 
Et c'est incontestable que Les oranges sont vertes ont troublé notre sérénité factice, nos calculs plats, nos escroqueries à l'Art. Nous nous dépêchons d'oublier leur fascinante apparition dans l'Arbre de Vie pour ne pas être atteint dans notre refus d'une autre réalité temporelle que celle imposée quotidiennement par nos ventres rétractés. N'oublions jamais que nous sommes tous de passage sur cette planète et que le Seul, l'Ultime engagement de l'Étre avec la Vie est d'atteindre la Transcendance, pour ne plus avoir à maudire notre existence de bêtes de somme.

Claude Gauvreau, par sa poésie dramatisée nous transhume, nous déifie en nous faisant percevoir toute la force, bien ou mal dirigée que l'on peut développer en chacun de nous (les tarés de naissance, ça n'existe pas, les tares nous viennent par l'évolution civilisatrice). Claude Gauvreau nous propose d'une manière visuelle et sonore (Mougnan dans Les oranges sont vertes) le dédoublement astral qui est le premier moyen de s'assumer dans l'univers. Son théâtre atteint les deux pôles de la poétique: le vécu et le devenir dans le présent confondus. Et le présent se consume pour atteindre la vision prophétique de l'homme déchu par la robotisation et l'efficacité exigée par ses congénères dans une optique systématisée. Batlam pourrait nous sauver, viendra-t-il ? en attendant "à bas, la phalange des branleurs".

Il faut une solide confiance pour aborder la formule théâtrale afin d'y traduire le cheminement de sa pensée, alors que le théâtre est une aventure périlleuse pour un poète où il ne lui est pas permis de s'appuyer uniquement sur sa création et son travail pour divulguer de nouvelles voies authentiques. Il doit convaincre le metteur en scène, les comédiens, le décorateur, les techniciens, les critiques, le public, etc. et pour cela subir des pressions de toutes sortes, des coupures de texte, des fignolages, des effets visuels collés sur l'image désirée. Le théâtre poétique est un art difficile à vivre et à mourir.

"Une vraie pièce de théâtre bouscule le repos des sens, libère l'inconscient comprimé, pousse à une sorte de révolte virtuelle et qui d'ailleurs ne peut avoir tout son prix que si elle demeure virtuelle, impose aux collectivités rassemblées une attitude héroïque et difficile." (Antonin Artaud)

Pourquoi le théâtre de Claude Gauvreau est-il si rude d'accès aux interprètes? au public? La première erreur est de penser qu'un langage personnalisé nuit à la communication... le processus à la mode étant de désapprendre à parler, à penser, à agir individuellement! «Sans hystérie collective, l'homme ne bouge pas" semble-t-on vouloir dire. Certains pensent que l'identification de l'individu se fera par l'unification d'une langue bornée; il faut surtout ne rien apprendre que l'on ne sache déjà, alors on élimine les mots qui suscitent la curiosité, l'éveil, l'attention - on tient à bâtardiser la langue en la réduisant à des données spécifiques, on nous fait passer de la mode maxi à la mode mini d'expression sonore et on prône le vote collectif qui élimine la minorité de ceux qui ne sont pas d'accord, on impose une manière d'être, d'agir, de parler, on lève les barrières et l'on dit à ceux qui n'obéissent pas

“parle comme nous'aut's ou ben j't'e casse la gueule» 
et l'on s'accroche à un mouvement politique pour couvrir sa retraite de lâche et de vaincu. Certains poètes ont préféré continuer leur route, solitaires sans échec et sans gloire car ils n'appartiennent plus au troupeau dirigé. Le poète sait qu'il peut par la science du langage recréer pour l'individu une repersonnalisation de son égo, en suscitant des possibilités d'évasion des contraintes par le rêve, le délire, les divagations spirituelles riches de liberté et d'innocence; le poète est un être habité, dangereux, qui nuit aux cadres établis de n'importe laquelle des sociétés étroitement structurées... le poète fait chambranler bien des murs, n'obéit pas à une masse ni n'impose une dictature ou autre discipline forcenée... le poète est un IMMENSE PROVOCATEUR qui abolit les portes, murs, barreaux, plafonds, planchers - il est l'instigateur de la GRANDE OUVERTURE, secouant les ombres, il attise le feu de la passion, joue avec la lumière, met à jour les cellules cervicales endormies et suspend le chatouillement du nombril hélas devenu pour plusieurs leur unique préoccupation.

Claude Gauvreau poète dérange nos normes, nos statuts, nos préjugés, nos visières, notre ignorance, notre paresse, notre absurdité à vouloir prolonger notre hébétude devant l'existence toquée que l'on mène. La continuité dans les recherches poussées à l'extrême de l'en-dedans (langage exploréen) nous enseigne la flagrance d'une exigence des plus rigoureuses dans la communication et l'expression de la parole... la parole de Claude Gauvreau est une ligne-flèche pointée vers l'infini avec un goût profond de l'absolu qui s'arrête à la masse lumière et le théâtre de Claude Gauvreau c'est l'âme québécoise assise sur un pic se confrontant avec l'homme préfabriqué québécois demeuré en bas qui n'ose pas se rendre compte qu'il pourrait grimper au-dessus de toutes les contingences qui l'assaillent - c'est aussi le cœur et l'esprit au paroxysme de l'entendement - c'est l'initiation aux cellules ouvertes par la condensation de l'imagination débordante et fabuleuse et nous serons très lents à comprendre que Claude Gauvreau fut le premier au Québec à réunir la réalité et le rêve, deux manières d'être inhérentes l'une à l'autre.

QUI EST BATLAM? m'ont demandé plusieurs étudiants... se peut-il que l'on n'ait pas compris... ou peut-être cela n'existe pas... n'a jamais existé... n'existera jamais... alors qui a inventé le mot «JUSTICE»? la justice et les frères justiciers qui vous assassinent à la fin du spectacle de Les oranges sont vertes ne vous ont-ils pas arrachés de vos chaînes? parfois je me demande quelle jouissance vous trouvez dans l'esclavage de votre pensée contrôlée, censurée, dirigée, amenuisée, asphyxiée?

BARIBEAU : Enfin! Allez-vous me dire? qui est ce Batlam? COCHEBENNE : Batlam, c'est Batlam. Entre-toi ça dans ta petite tête...

Ce texte n'est ni extrait ni résumé de ma prochaine publication Claude Gauvreau, le cygne où il est question

de la souffrance de Claude Gauvreau

externe

et de son sourire intérieur 
de la folie de Claude Gauvreau

vulnérable

et de sa lucidité ample à éclater

de la poésie exploréenne de Claude Gauvreau

et de sa quête de la lumière

de la dramaturgie pataphysique de Claude Gauvreau

et de sa transcendance démesurée

des mots à dire à Claude Gauvreau

et des mots dits

de sa clarté douce opérante

et des défaitistes niant les constantes de la réalité

de la haute solidarité de Claude Gauvreau

et du lion qu'on repousse dans sa cage

- il n'en est que l'embryon qui me servit à faire ressurgir dans ma mémoire, certains faits certaines paroles certaines oppressions certains échanges qui livrés à votre connaissance témoigneront plus de l'homme et de son œuvre que n'importe laquelle des propagandes, car pour moi

doué d'ailes puissantes

plutôt que de serres fortes

qu'il demeure l'aigle

pour les effraies et les alouates

pour moi il est le cygne

que l'on entend longtemps mourir

sans l'entendre vivre

Janou Saint-Denis

1. Premier jet-résumé d'un témoignage-essai à venir, sorte de chronique vécue de la période présumée morte des années 50-60, commencé à formuler en janvier 74 et qui donnera un livre intitulé: Claude Gauvreau, le cygne à paraître bientôt aux Presses de l'Université du Québec en collaboration avec les éditions du Noroît.

2. Public d'étudiants, de jeunes créateurs, d'infirmières et de voyous sans titre... 